J. Nonlinear Sci. Appl. 1 (2008), no. 1, $31-35$

The Journal of $\mathbf{N}_{\text {onlinear }} \mathbf{S}_{\text {ciences and its }}$ A pplications

http://www.tjnsa.com

\title{
COMMON FIXED POINT THEOREM IN PROBABILISTIC QUASI-METRIC SPACES
}

\author{
A.R. SHABANI ${ }^{1, *}$, S. GHASEMPOUR ${ }^{2}$
}

\begin{abstract}
In this paper, we consider complete probabilistic quasi-metric space and prove a common fixed point theorem for $R$-weakly commuting maps in this space.
\end{abstract}

\section{INTRODUCTION}

Menger introduced the notion of a probabilistic metric spaces in 1942 and, since then, the theory of probabilistic metric spaces has developed in many directions, especially, in nonlinear analysis and applications [5]. The idea of Menger was to use distribution functions instead of nonnegative real numbers as values of the metric.

Recently, Pant introduced the notion of $R$-weak commutativity of mappings in metric spaces and proved some common fixed point theorems.

In this paper, we define $R$-weak commutativity of mapping in probabilistic quasi-metric spaces and prove the probabilistic version of Pant's theorem. In the sequel, we shall adopt usual terminology, notation and conventions of the theory of probabilistic metric spaces, as in [1, 5].

Throughout this paper, the space of all probability distribution functions (briefly, d.f.'s) denotes $\Delta^{+}=\{F: \mathbf{R} \cup\{-\infty,+\infty\} \longrightarrow[0,1]: F$ is left-continuous and non-decreasing on $\mathbf{R}, F(0)=0$ and $F(+\infty)=1\}$ and the subset $D^{+} \subseteq \Delta^{+}$is the set $D^{+}=\left\{F \in \Delta^{+}: l^{-} F(+\infty)=1\right\}$. Here $l^{-} f(x)$ denotes the left limit of the function $f$ at the point $x, l^{-} f(x)=\lim _{t \rightarrow x^{-}} f(t)$. The space $\Delta^{+}$is partially ordered by the usual point-wise ordering of functions, i.e., $F \leq G$ if and only if

Date: Received: 2 March 2008.

* Corresponding author.

2000 Mathematics Subject Classification. Primary 54E70; Secondary 54H25.

Key words and phrases. Probabilistic metric spaces; quasi-metric spaces; fixed point theorem; $R$-weakly commuting maps; triangle function. 
$F(x) \leq G(x)$ for all $x$ in $\mathbf{R}$. The maximal element for $\Delta^{+}$in this order is the d.f. given by $\varepsilon_{0}=0$, if $x \leq 0$ and $\varepsilon_{0}=1$, if $x>0$

We assume that $\Delta^{+}$is metrized by the Sibley metric $d_{S}$, which is the modified Lévy metric [4, 6]. If $F$ and $G$ are d.f.'s and $h \in(0,1]$, let $(F, G ; h)$ denote the condition

$$
F(x-h)-h \leq G(x) \leq F(x+h)+h
$$

for all $x$ in $(-1 / h, 1 / h)$. Then the modified Lévy metric (Sibley metric) is defined by

$$
d_{S}(F, G):=\inf \{h: \operatorname{both}(F, G ; h) \text { and }(G, F ; h) \text { hold }\} .
$$

For any $F$ in $\Delta^{+}$,

$$
\begin{aligned}
d_{S}\left(F, \varepsilon_{0}\right) & =\inf \left\{h:\left(F, \varepsilon_{0} ; h\right) h o l d s\right\} \\
& =\inf \left\{h: F\left(h^{+}\right)>1-h\right\}
\end{aligned}
$$

and, for any $t>0$,

$$
F(t)>1-t \Longleftrightarrow d_{S}\left(F, \varepsilon_{0}\right)<t .
$$

It follows that, for every $F, G$ in $\Delta^{+}$,

$$
F \leq G \Longrightarrow d_{S}\left(G, \varepsilon_{0}\right) \leq d_{S}\left(F, \varepsilon_{0}\right) .
$$

A triangle function is a binary operation on $\Delta^{+}$, namely, a function $\tau: \Delta^{+} \times$ $\Delta^{+} \longrightarrow \Delta^{+}$that is associative, commutative, nondecreasing and which has $\varepsilon_{0}$ as unit, i.e., for all $F, G, H \in \Delta^{+}$,

$$
\begin{aligned}
\tau(\tau(F, G), H) & =\tau(F, \tau(G, H)), \\
\tau(F, G) & =\tau(G, F), \\
F \leq G & \Longrightarrow \tau(F, H) \leq \tau(G, H), \\
\tau\left(F, \varepsilon_{0}\right) & =F .
\end{aligned}
$$

Continuity of a triangle function means continuity with respect to the topology of weak convergence in $\Delta^{+}$. Typical continuous triangle function is $\tau_{T}(F, G)(x)=$ $\sup _{s+t=x} T(F(s), G(t))$. Here $T$ is a continuous $t$-norm, i.e., a continuous binary operation on $[0,1]$ that is commutative, associative, nondecreasing in each variable and has 1 as identity. Two typical examples of continuous $t$-norm are $\pi(a, b)=a b$ and $W(a, b)=\max (a+b-1,0)$.

Definition 1.1. A Probabilistic Quasi-Metric (briefly, PQM) space is a triple $(X, \mathcal{F}, \tau)$, where $X$ is a nonempty set, $\tau$ is a continuous triangle function, and $\mathbf{F}$ is a mapping from $X \times X$ into $D^{+}$such that, if $F_{p, q}$ denotes the value of $\mathcal{F}$ at the pair $(p, q)$, the following conditions hold for all $p, q, r$ in $X$ :

(PQM1) $F_{p, q}=F_{q, p}=\varepsilon_{0}$ if and only if, $p=q$;

(PQM2) $F_{p, q} \geq \tau\left(F_{p, r}, F_{r, q}\right)$ for all $p, q, r \in X$.

Theorem 1.2. ([5) If $(X, \mathcal{F}, \tau)$ is a PQM space with $\tau \geq \tau_{W}$ and function $\beta$ is defined by

$$
\beta(p, q)=d_{S}\left(F_{p, q}, \varepsilon_{0}\right) .
$$

Then $\beta$ is a quasi-metric. 
Definition 1.3. Let $(X, \mathcal{F}, \tau)$ be a PQM space.

(1) A sequence $\left\{p_{n}\right\}_{n}$ in $X$ is said to be strongly convergent to $p$ in $X$ if, for every $\lambda>0$, there exists positive integer $N$ such that $F_{p_{n}, p}(\lambda)>1-\lambda$ whenever $n \geq N$.

(2) A sequence $\left\{p_{n}\right\}_{n}$ in $X$ is called strong right (left) Cauchy sequence [3] if, for every $\lambda>0$, there exists positive integer $N$ such that $F_{p_{n}, p_{m}}(\lambda)>1-\lambda$ whenever $n \geq m \geq N(m \geq n \geq N)$.

(3) A PQM space $(X, \overline{\mathcal{F}}, \tau)$ is said to be strong right (left) complete in the strong topology if and only if every strong right (left) Cauchy sequence in $X$ is strongly convergent to a point in $X$.

Theorem 1.4. ([5]) Let $(X, \mathcal{F}, \tau)$ be a PQM space and let $\left\{p_{n}\right\}$ be a sequence in $X$. Then $p_{n} \rightarrow p$ if and only if $d_{S}\left(F_{p_{n}, p}, \varepsilon_{0}\right) \rightarrow 0$ if and only if $\beta\left(p_{n}, p\right) \rightarrow 0$. Similarly, $\left\{p_{n}\right\}$ is a strong right (left) Cauchy sequence if and only if for every $\epsilon>0$ there exists positive integer $N$ such that

$$
\beta\left(p_{n}, p_{m}\right)<\epsilon
$$

whenever $n \geq m \geq N(m \geq n \geq N)$.

Theorem 1.5. ([4]) If $\tau$ is a continuous triangle function and $\left\{p_{n}\right\}$ and $\left\{q_{n}\right\}$ are sequences such that $p_{n} \rightarrow p$ and $q_{n} \rightarrow q$, then $\lim _{n \rightarrow \infty} F_{p_{n}, q_{n}}=F_{p, q}$.

\section{Main Results}

Definition 2.1. Let $f$ and $g$ be maps from a PQM space $(X, \mathcal{F}, \tau)$ into itself. The maps $f$ and $g$ are said to be weakly commuting if

$$
F_{f g x, g f x} \geq F_{f x, g x}
$$

for each $x$ in $X$.

Definition 2.2. Let $f$ and $g$ be maps from a PQM space $(X, \mathcal{F}, \tau)$ into itself. The maps $f$ and $g$ are said to be $R$-weakly commuting of type $\left(A_{f}\right)$ if there exists a positive real number $R$ such that

$$
F_{f g x, g g x}(t) \geq F_{f x, g x}(t / R)
$$

for each $x \in X$ and $t>0$.

Weak commutativity implies $R$-weak commutativity in PQM space. However, $R$-weak commutativity implies weak commutativity only when $R \leq 1$.

Theorem 2.3. Let $(X, \mathcal{F}, \tau)$ be a left complete PQM space in which $\tau \geq \tau_{W}$ and let $f, g$ be $R$-weakly commuting self-mappings of $X$ satisfying the following conditions:

(i) $f(X) \subseteq g(X)$;

(ii) $f$ or $g$ is continuous;

(iii) for all $x, y \in X$,

$$
F_{f x, f y} \geq C\left(F_{g x, g y}\right)
$$

where $C: D^{+} \longrightarrow D^{+}$is a continuous function such that $C(F)>F$ for each $F \in D^{+}$with $F<\varepsilon_{0}$.

Then $f$ and $g$ have a unique common fixed point in $X$. 
Proof. Let $x_{0}$ be an arbitrary point in $X$. By (i), choose a point $x_{1}$ in $X$ such that $f x_{0}=g x_{1}$. In general, choose $x_{n+1}$ such that $f x_{n}=g x_{n+1}$. Then we have

$$
\begin{aligned}
F_{f x_{n}, f x_{n+1}} & \geq C\left(F_{g x_{n}, g x_{n+1}}\right)=C\left(F_{f x_{n-1}, f x_{n}}\right) \\
& >F_{f x_{n-1}, f x_{n}}
\end{aligned}
$$

Thus $\left\{F_{f x_{n}, f x_{n+1}}\right\}$ is increasing sequence in $D^{+}$. Therefore, it converges to a limit $G \leq \varepsilon_{0}$. We claim that $G=\varepsilon_{0}$. For, if $G<\varepsilon_{0}$ on making $n \longrightarrow \infty$ in the above inequality, we get $G \geq C(G)>G$, which is a contradiction. Hence $G=\varepsilon_{0}$, i.e.,

$$
\lim _{n \rightarrow \infty} F_{f x_{n}, f x_{n+1}}=\varepsilon_{0} .
$$

By Theorem 1.4, there exists $n_{0} \in \mathbf{N}$ such that, for every $n \geq n_{0}$,

$$
\beta\left(f x_{n}, f x_{n+1}\right) \leq \frac{1}{2^{n}} .
$$

Now, for every $m, n \in \mathbf{N}$ with $m>n$, we have

$$
\beta\left(f x_{n}, f x_{m}\right) \leq \sum_{j=n}^{m} \beta\left(f x_{j}, f x_{j+1}\right) \leq \sum_{j=n}^{m} \frac{1}{2^{j}} \longrightarrow 0
$$

as $m, n \rightarrow \infty$. Thus, by Theorem 1.4. $\left\{f x_{n}\right\}$ is left Cauchy sequence and, by the left completeness of $X,\left\{f x_{n}\right\}$ converges to $z \in X$. Also $\left\{g x_{n}\right\}$ converges to $z$ in $X$. Let us suppose that the mapping $f$ is continuous. Then $\lim _{n \rightarrow \infty} f f x_{n}=f z$ and $\lim _{n \rightarrow \infty} f g x_{n}=f z$. Further, since $f$ and $g$ are $R$-weakly commuting, we have

$$
F_{f g x_{n}, g f x_{n}}(t) \geq F_{f x_{n}, g x_{n}}(t / R) .
$$

On letting $n \rightarrow \infty$ in the above inequality, we have $\lim _{n \rightarrow \infty} g f x_{n}=f z$ by Theorem 1.5 .

We now prove that $z=f z$. Suppose $z \neq f z$. Then $F_{z, f z}<\varepsilon_{0}$. By (iii),

$$
F_{f x_{n}, f f x_{n}} \geq C\left(F_{g x_{n}, g f x_{n}}\right) \text {. }
$$

On letting $n \rightarrow \infty$ in the above inequality, we have

$$
F_{z, f z} \geq C\left(F_{z, f z}\right)>F_{z, f z}
$$

which is a contradiction. Therefore, $z=f z$. Since $f(X) \subseteq g(X)$, we can find a point $z_{1} \in X$ such that $z=f z=g z_{1}$. Now,

$$
F_{f f x_{n}, f z_{1}} \geq C\left(F_{g f x_{n}, g z_{1}}\right) .
$$

Taking limit as $n \rightarrow \infty$, we have

$$
F_{f z, f z_{1}} \geq C\left(F_{f z, g z_{1}}\right)=\varepsilon_{0}
$$

since $C\left(\varepsilon_{0}\right)=\varepsilon_{0}$, which implies that $f z=f z_{1}$, i.e., $z=f z=f z_{1}=g z_{1}$. Also, for any $t>0$,

$$
F_{f z, g z}(t)=F_{f g z_{1}, g f z_{1}}(t) \geq F_{f z_{1}, g z_{1}}(t / R)=\varepsilon_{0}(t / R)=1
$$

which again implies that $f z=g z$. Thus $z$ is a common fixed point of $f$ and $g$.

Now, to prove the uniqueness of the common fixed point $z$, let $z^{\prime} \neq z$ be another common fixed point of $f$ and $g$. Then $F_{z, z^{\prime}}<\varepsilon_{0}$ and

$$
F_{z, z^{\prime}}=F_{f z, f z^{\prime}} \geq C\left(F_{g z, g z^{\prime}}\right)=C\left(F_{z, z^{\prime}}\right)>F_{z, z^{\prime}},
$$


which is contradiction. Therefore, $z=z^{\prime}$, i.e., $z$ is a unique common fixed point of $f$ and $g$.

\section{REFERENCES}

[1] I. Beg, Fixed points of contractive mappings on probabilistic Banach spaces, Stoch. Anal. Appl. 19 (2001), 455-460.

[2] S. S. Chang, B. S. Lee, Y. J. Cho, Y. Q. Chen, S. M. Kang and J. S. Jung, Generalized contraction mapping principles and differential equations in probabilistic metric spaces, Proc. Amer. Math. Soc. 124 (1996), 2367-2376. 1

[3] I. L. Reilly, P. V. Subrahmanyam and M. K. Vamanamurthy, Cauchy sequences in quasipseudo- metric spaces, Monatsh. Math. 93 (1982), 127-140.

[4] B. Schweizer and A. Sklar, Probabilistic Metric Spaces , (Elsevier North Holand, New York, 1983).

[5] B. Schweizer, H. Sherwood and R. M. Tardiff, Contractions on PM-space examples and counterexamples, Stochastica 1(1988), 5-17.

[6] D. A. Sibley, A metric for weak convergence of distribution functions, Rocky Mountain J. Math. 1 (1971), 427-430. 1.3

1 , 1.5

$1,1.2,1.4$

1

${ }^{1}$ Department of Mathematics, Imam Khomaini Mritime University of Nowshahr NOWSHAHR, IRAN

E-mail address: a.r.shabai@yahoo.com

2 Department of Mathematics, Payam noor University, Amol, Iran

E-mail address: ghasempour_pnu@yahoo.com 